\title{
Princípios éticos como norteadores no cuidado domiciliar
}

\author{
Ethical principles as a home care guide
}

Letícia Rosa Santos ${ }^{1}$

Casandra Genoveva Rosales M artins Ponce de Leon ${ }^{2}$

Silvana Schwerz Funghetto ${ }^{2}$

${ }^{1}$ M inistério da Saúde. Esplanada dos M inistérios, Bloco G, 9 on andar, Asa Sul. 70058-900 Brasília DF. tenenteleticia@yahoo.com.br ${ }^{2}$ U niversidade de Brasília.

\begin{abstract}
H omecareisa health carestrategy that aims to emphasize the autonomy of the patient as well as to enhance their functional skills in their home. The aim is to reflect the observance of ethical principles in home care, in the form of home assistance. The study is exploratory and descriptive, addressing a critical review of the literature, according to the analysis of Bardin. H ome care is becoming indispensablein reducing hospital costs, hospitalization, number of hospitalizations and number of clinical complicationsm as well as increased family participation in patient care, providing a better quality of life. However, it is necessary to take into consideration the purpose and quality of care delivered, analyzing it from the perspective of ethical principles in order not to make this kind of care only beneficial to one party, but a common good to all subjects involved in this care.
\end{abstract}

Key words Nursing, Ethics, Home care
Resumo 0 cuidado domiciliar é uma estratégia de atenção à saúde que visa enfatizar a autonomia do paciente, bem como realçar habilidades funcionais em seu contexto domiciliar. 0 objetivo é refletir a observância dos princípios éticos no cuidado domiciliar, na modalidade de assistência domiciliar. 0 estudo é do tipo exploratório descritivo, contemplando uma revisão crítica da bibliografia, segundo a análise de conteúdo de Bardin. 0 cuidado domiciliar vem se tornando imprescindível para reduzir custos hospitalares, permanência hospitalar, número de reinternações e o número de complicações clínicas, bem como 0 aumento da participação da família no cuidado ao paciente, proporcionando uma melhor qualidade de vida. Entretanto, é necessário levar em consideração a finalidade equalidade desta assistência prestada, analisando-a sob a ótica dos princípios éticos, de modo a não tornar este tipo de cuidado benéfico apenas para uma das partes, e sim um bem comum a todos os sujeitos envolvidos nesse cuidado.

Palavras-chave Enfermagem, Ética, Cuidado domiciliar 


\section{Introdução}

Atualmente, o cuidado de enfermagem domiciliar está em pauta, em decorrência, principalmente, de necessidades sociais e de saúde da população. A atenção no domicílio agrega mais que um "tratamento médico residencial padronizado", pois real ça as habilidades funcionais do paciente, enfatizando sua autonomia ${ }^{1}$.

0 foco deste estudo reside no que se refere à modalidade de assistência domiciliar. Tal modalidadeengloba situações de cui dado intermitente por um dano agudo ou agravo de longa duração, envolvendo ações educativas e/ ou realização de procedimentos que visam à redução do dano eà prevenção.

Vários os fatores justificam a tendência à implantação de serviços de cuidado domiciliar, tais como diminuição de complicações clínicas, reinternações, hipertrofia dos serviços de emergência, custos hospitalares e óbitos, bem como uma maior participação da família no cuidado². Entretanto, para que tal estratégia seja benéfica para todos os seguimentos da sociedade, é necessário relacionar suas justificativas à luz dos princípios éticos. Existem várias perspectivas de abordagem ética, as quais consideram diferentes características e modelos explicativos, desenvolvidos a partir de diferentes referenciais teóricos no contexto da tomada de decisão moral. Um dos modelos mais difundidos na área da saúde é o principialista, defendido por Tom Beauchamp e James Childress ${ }^{3}$.

A abordagem dos quatro princípios de ética biomédica de Beauchamp e Childress ${ }^{3}$ ou principialismo, como ficou conhecida mundialmente, se orienta por quatro princípios: o princípio da beneficência diz respeito à obrigação moral deagir em benefício dos outros, ou seja, fazer ou promover o bem, assim como impedir e eliminar males ou danos; o princípio da não maleficiência, que preconiza acima de tudo não causar mal ou dano as pessoas; o princípio dajustiça, obrigação ética de tratar cada pessoa de acordo com o queé certo e adequado e dar o que lhe é devido; e, por fim, o princípio da autonomia, condição deliberdadeou independência para a escolha dealternativas sem qualquer tipo de controle ea capacidade do indivíduo agir intencionalmente.

0 principialismo teve grande destaque na década de setenta e até hoje é adotado; porém, tem sido criticado por aplicar os princípios de modo essencialmenteobjetivo eracional. Embora existam diversas abordagens das questões éti- cas que divergiram em decorrência das diferentes visões de mundo e da evolução dos conhecimentos, algumas noções ainda perduram, como a de agir de acordo com o bem. Os princípios constituem um referencial ético concreto, que apresenta um valor metodológico importante, que permite reduzir incertezas no campo das ações biomédicas.

Frente a este contexto, faz-senecessário refletir a observância dos princípios éticos no cuidado domiciliar, na modalidade de assistência domiciliar.

\section{M étodo}

Trata-se de um estudo exploratório descritivo, caracterizado por uma revisão crítica da bibliografia sobre como o cuidado domiciliar discute os princípios éticos à luz da teoria de Beauchamp e Childress ${ }^{3}$. Para tanto, realizou-se uma busca de publicações indexadas nas seguintes bases de dados: LILACS, MEDLINE, SciELO, BDENF e CAPES, utilizando-se como descritor controlado o cuidado domiciliar (na modalidade assistência domiciliar), foco deste estudo. Posteriormente, as fontes encontradas foram confrontadas com teoria dos quatro princípios para que fossem selecionadas para a análise final.

A localização dos documentos ocorreu por intermédio de comutação bibliográfica, avesso de acervo disponível online e acervos particulares de pesquisadores da área.

Os estudos foram analisados com basena técnica de análise de conteúdo de Bardin ${ }^{4}$, em que foram elaboradas três categorias preestabelecidas. Assim, respondeu-se às seguintes questões:

- Os profissionais de saúde respeitam o princípio da beneficência enão-maleficência ao delegarem suas funções aos cuidadores informais, e estes têm condições de atuarem como provedores do cuidado no domicílio?

- Estamos oferecendo o cuidado domiciliar obedecendo ao princípio de justiça, como uma modalidade, ou impondo como uma alternativa mais conveniente para a instituição e/ou sistema de saúde?

- 0 cuidado domiciliar oportuniza a autonomia do paciente e da família no que se refere a esta estratégia assistencial?

Os princípios éticos foram respeitados, procurando proteger os direitos devido à utilização dos referenciais bibliográficos, para a construção deste estudo. 


\section{Resultados}

Os temas relacionados às categorias foram relacionados no Quadro 1.

\section{Beneficência e não-mal eficência}

Observa-se que os serviços de atendimento domiciliário no Brasil estiveram quase sempre ligados à área da Saúde Pública, visando à promoção da saúdee à prevenção de doençaš2. Mundialmente, essa estratégia assistencial está difundindo-se rapidamente, devido à questão econômica, sendo esta um dos três fatores mais importantes no seu crescimento ${ }^{5}$.

Diante desse contexto, as implementações de programas de cuidado domiciliares precisam privilegiar o cuidado humano no seu contexto natural, bem como fatores que justificam tal implementação, que se referem à transição do perfil demográfico e epidemiológico da população brasileira, ocasionando um aumento de doenças crônico-degenerativas acompanhadas do risco de complicações e do nível de dependência; inadequação do atual sistema de saúde, em que questões não emergenciais poderiam ser resolvidas em nível de atenção básica, bem como a existência de uma boa articulação com os demais níveis de atenção, fato este que denota uma importante falha no sistema de referência e contrarreferência, sendo isto percebido através da hipertrofia em emergências; adoção do Programa de Saúde da Família (PSF), que tem a maio- ria de suas ações em nível primário de atenção, sendo necessário complementar as ações através do Programa de Saúde Domiciliar, com a enfermeira implementando ações de promoção à saúde, sendo o foco na educação em saúde; a família passa por transformações na sua organização de extensa para nuclear - em que as funções de cuidado são, paulatinamente, compartilhadas com instituições criadas para esse fim ${ }^{2,3,6,7}$.

Vários estudos apontam que o cuidado domiciliar diminui o tempo médio de internação hospitalar, reduz o número de reinternações, reduz custos de atenção à saúde, aumenta a ade rência ao tratamento do paciente sob assistência domiciliar. Além disso, nota-se a melhora da qualidade de vida do paciente e familiar, desenvolve-se como um novo instrumento de ensino aos graduandos e abertura de um novo campo de ação em pesquisa. Portanto, tal estratégia proporciona maior conscientização ao paciente e cuidador do quadro patológico atual e, consequentemente, maior autonomia no tratamento, bem como as prioridades de cuidado ao paciente no domicílio são muito diferentes do cuidado realizado no hospital $3,5,8-11$.

Entretanto, o despreparo do cuidador pode também trazer sérios prejuízos ao paciente, re sultando inclusive em frequentes hospitalizações ${ }^{12}$. Além disso, o despreparo pode gerar ansiedade e maior desgaste físico, ocasionando situações derisco para ambos ${ }^{13,14}$. Estudo demonstra que $67,5 \%$ dos cuidadores relataram ter dificuldades para realizar o cuidado. As dificuldades

Quadro 1. Categorias e temas.

\begin{tabular}{|c|c|}
\hline Categorias & Temas \\
\hline Beneficência e não-maleficência & $\begin{array}{l}\text { - Justificativa do cuidado domiciliar } \\
\text { - Ações dos profissionais e dos cuidadores informais } \\
\text { - Condições da família de atuar como cuidadores } \\
\text { - Condições dos profissionais e das instituições para atuar } \\
\text { - Vínculo do sistema de saúde com a família }\end{array}$ \\
\hline Justiça & $\begin{array}{l}\text { - Definição e modalidade de cuidado domiciliar } \\
\text { - Objetivos do cuidado domiciliar } \\
\text { - Objetivos da família } \\
\text { - Objetivos do paciente } \\
\text { - Objetivos da instituição }\end{array}$ \\
\hline Autonomia & $\begin{array}{l}\text { - Processo de decisão } \\
\text { - Relação familiar } \\
\text { - Relação da família x equipe }\end{array}$ \\
\hline
\end{tabular}


manifestadas pelos cuidadores não expressam apenas dificuldades específicas a atividades referentes ao cuidado, mas relacionadas à dinâmica familiar e aos sentimentos dos cuidadores ${ }^{15,16}$. Para cuidar deum adulto dependente, énecessário ter condicionamento físico capaz de dar conta de tarefas pesadas, como a locomoção e outras, além derecuperar-se rapidamente, por exemplo, de uma noite mal dormida.

Quanto ao aspecto ético-legal, é o mesmo tanto na instituição como no domicílio. Porém, os profissionais devem estar cientes e conscientes quanto à perda do manto corporativo hospitalar queatéhoje encobriu o abuso depoder, omissões, negligências e imperícias ${ }^{6}$.

As ações do Programa de Saúde da Família (PSF), na sua maioria, contemplam o nível primário de aten ção, sendo necessário complementar as ações através do Programa de Saúde Domiciliar, com a enfermeira implementando ações de promoção à saúde, tendo o foco na educação em saúde 6 .

Para o cuidado domiciliar acontecer, deve-se iniciar o planejamento no ambiente hospitalar, em que a família/cuidador é submetida às limitações que porventura podem surgir; assi $m$, permite-se gradativamente garantir um cuidado adequado no domicílio. 0 acompanhamento do paciente no domicílio pode ser diário, semanal, quinzenal, mensal, de acordo com a sua situação clínica ${ }^{2,9}$.

Alguns estudos apontam que o planejamento do cuidado domiciliar inicialmenteéuma proposta do médico e enfermeira, os quais decidem se o paciente tem condições de receber esse tipo de acompanhamento no domicílio. No entanto, são poucos os estudos que destacam a participação da família/cuidador no momento da decisão sobre a utilização dessa estratégia assistencial ${ }^{6,17}$.

$\mathrm{N}$ esse momento, deve-se considerar a organização familiar e comunitária para integrar-se o sistema de cuidado do profissional de saúde com o sistema de cuidado popular exercido pelo grupo familiar ou rede de apoio social do paciente. Assim, a parceria entre profissionais e pessoas que cuidam deve possibilitar a sistematização de tarefas a serem realizadas no domicílio, privilegiando ações de promoção à saúde, prevenção de incapacidades, manutenção da capacidade funcional, evitando, na medida do possível, hospitalizações, asilamentos, outras formas de segregação e isolamento $0^{3,7,18}$.

0 espaço domiciliar permitelidar com situações novas, proporcionando constante aprendizado ao profissional de saúde. Porém, tal estra- tégia coloca o enfermeiro numa posição que exige capacidade de decisão eautonomia, peranteo pacienteque está sob sua responsabilidade técnica e legal . Frente a este contexto, a falta de capacitação em assistência domiciliar gera dificuldades quanto ao exercício profissional, exercício da autonomia, habilidades de relacionamento interpessoal, bem como de relação de ajuda ${ }^{5,6}$.

Em relação ao acesso e vínculo, autores do Estados Unidos destacam que o cuidado domiciliar deve assegurar acesso a todos os serviços de saúde do país, independente de onde o paciente resida. Na Espanha, o cuidado domiciliar eo hospital devem estar organizados adequadamente, financiados e integrados para prover tal atendimento de forma contínua e eficaz. No Brasil, o cuidado domiciliar seria a interface entre a Unidade Básica de Saúde e a rede hospitalar, imple mentando terapias no próprio domicílio e, quando necessário, nos locais para cuidados de aten-

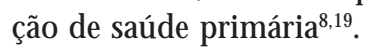

\section{Justiça}

Os custos relacionados ao sistema de saúde são sempre questões preocupantes para os envolvidos com a área. Estudos mostram que as intervenções na assistência domiciliária equivalem a aproximadamente um terço do custo das intervenções realizadas em ambiente hospitalar, sendo seu custo médio diário de pacienteem cuidado domiciliar de $\mathrm{R} \$ 6,48$, bem inferior ao dos pacientes internados ${ }^{2,20}$.

0 modelo de cuidado domiciliar apresenta inúmeras vantagens para as instituições e sistemas de saúde; entre elas, reduzir o custo do tratamento, diminuir o número de diárias, baixar os custos dos serviços de saúde ( gastos com instalações e equipamentos), não pagar serviços cobrados por pacotes (como taxa de aplicações, soro, etc. $)^{2,18,21}$.

Esta modalidade de cuidado nas instituições permite uma maior rotatividade de seus leitos, otimizando a dinâmica de ocupação dos mesmos. A transferência dos pacientes crônicos e convalescentes para o cuidado domiciliar libera o leito hospitalar, abrindo espaço para pacientes instáveis que precisam realmente de UTI, cirurgias, politraumatizados e outras enfermidades agudas, ampliando assim a oferta de leitos, diminuindo a média de permanência hospitalar, as reinternaçõese, consequentemente, os custos assistenciais. Além disso, o paciente crônico gera menor receita em sua estada/dia para as instituições e sistemas de saúde, o que o tornaria um 
paciente ideal para ser indicado ao cuidado domiciliar ${ }^{22}$.

A otimização de leitos acarretará uma maior margem de lucro pelo fato da instituição não precisar elevar o seu efetivo de pessoal, mas permitirá capacitá-lo melhor com treinamentos mais específicos. Por sua vez, o dinheiro queseria destinado a obras de expansão poderia ser canalizado para a melhoria do atendimento, aquisição de equipamentos mais modernos e outras prioridades antes não previstas ${ }^{8,20}$.

A implantação de serviços de saúde domiciliar pode ser a estratégia que possibilitará um maior aproveitamento dosleitoshospitalares, oferecendo serviços de saúde ao público com maior eficiência eeconomia possíveis, reduzindo custos através da diminuição tanto do tempo das estadas nos hospitais quanto dos índices de internação hospitalar ${ }^{23}$.

Sem exceção, todos os modelos de saúde visam baixar o custo, aumentar a produtividade dos profissionais e aumentar o benefício para a clientela. Os custos gerados por essa dependência são tão grandes quanto 0 investimento de dedicar um membro da família ou um cuidador para ajudar continuamente uma pessoa que, muitas vezes, irá viver mais dez ou vinte anos. Sendo assim, esta modalidade de cuidado não poderá ter como única finalidade baratear custos e transferir responsabilidades ${ }^{20}$.

É importanteressaltar, no entanto, queisso não significa que a assistência domiciliária seja "barata", mas que, comparativamente ao sistema hospitalar, seu custo é significativamente inferior ${ }^{2,24}$.

O cuidado domiciliar também visa benefícios para os clientes, sendo estetipo deassistência uma forma de, na atual conjuntura de nosso sistema de saúde, sobrecarregado e sem recursos suficientes para a presente demanda de clientes, garantir a continuidade da assistência com o apoio de uma equipe de saúde interdisciplinar e viabilizar ao cliente os recursos terapêuticos e a assistência profissional que teria no hospital8,23,25,26.

O cuidado domiciliar visa melhorar a qualidade de vida e propiciar acompanhamento domiciliário dos pacientes crônicos e daqueles nos quais já foram esgotadas todas as possi bilidades de tratamento especializado. Essa assistência engloba a visitação domiciliar e cuidados domiciliares que vão desde 0 fornecimento de equipamentos, atéações terapêuticas mais complexas ${ }^{3,18}$.

E, como salientado, a redução do tempo de internação e reinternações não traz somente vantagens para as instituições no que tange aos custos, mas também aos indivíduos que correm menos riscos em consequência das hospitalizações, e propicia a utilização dos leitos por um número maior de pessoas, ampliando a oferta de leitos, ou seja, agiliza a liberação de leitos da unidade hospitalar destinados a doentes passíveis de hospitalização ou a portadores de casos mais graves ${ }^{6}$.

Diante dos custos hospitalares elevados e do baixo benefício resultantes das internações, os cuidados secundários estão sendo deslocados para os ambulatórios e para o domicílio do cliente. Poisa manutenção do atual modelo centrado na hospitalização significará criar uma situação de superlotação das unidades hospitalares, ocupadas por doentes idosos, com doenças crônico-degenerativas, aumentando os custos da assistência à saúde e comprometendo a qualidade do atendimento $0^{5,6,8,20}$.

A assistência domiciliar é um prolongamento do tratamento hospitalar e pode permitir a alta precoce dos doentes internados, diminuindo seu tempo depermanênciae, em decorrência, ampliando a capacidade de internação, suprindo o problemas de carência de vagas ${ }^{11,23,27}$.

0 atual contexto econômico esocial, com um aumento crescente da demanda dos serviços de saúde, necessita de um modelo de atenção à saúde que otimize os recursos escassos. Desta forma, o cuidado domiciliar surge como modelo harmônico com a economia vigente eque propicia uma ad equada assistência às necessidades dos usuários $8,18,20$.

\section{Autonomia}

A tomada de decisão quanto à permanência em casa ou no hospital do paciente pode ocorrer dediversas formas: por indicação médica ${ }^{3,8,28}$, um trabalho da equipe interdisciplinar no qual se realiza a discussão de casos em conjunto eas decisões são conhecidas por toda equipe ${ }^{23}$, ou em conjunto, num processo dinâmico entre a família os profissionais de saúde e o paciente ${ }^{3,5,22}$.

Outro estudo aponta questões importantes quanto às formas de indicação dos pacientes para este tipo de assistência. A seleção pode ocorrer de diversas formas; além da já citada decisão prévia deum integrante da equipedesaúde, existem ainda a sobrecarga da equipe ou envio de pacientes "pesados e incômodos" para as instituições hospitalares ${ }^{8}$, não existindo nestas últimas opções quaisquer critérios "benéficos" para o cliente.

Com relação à compreensão sobre a assistência domiciliar à saúde, observa-se que os profissionais podem considerá-la sob duas perspec- 
tivas: a de uma prática hierarquizada e impositiva, na qual são destacados os aspectos envolvidos diretamente com a dimensão biológica do adoecimento e a facilitação do atendimento; e a perspectiva relacional e interativa, como forma de atenção diferenciada e de práticas inovadoras de construção da saúde ${ }^{30}$.

$\mathrm{Na}$ Europa, a forma de financiamento determina quem toma a decisão de encaminhar os doentes para assistência domiciliar. Nos serviços "públicos" , o acesso independe do encaminhamento do médico. János que são financiados pela rede privada de saúde, a questão se inverte e a indicação médica passa a ser pré-requisito. No Brasil, não existeum padrão preestabelecido, mas há um predomínio daindicação médica, uma vez quea Lei ${ }^{\circ} 10.424$ de $2002^{31}$ e a Portaria n ${ }^{\circ} 2.421$ de $1998^{32}$ já colocam as diretrizes para o funcionamento do home caree como os serviços de saúde atendem os seus clientes. Assim, é necessário que o médico indique e solicite a internação ou assistência domiciliar para esse paciente ao serviço de home care. No setor privado, os casos são detectados pelos médicos assistentes ou médicos auditores, que identificam situações de internações prolongadas ou de reinternações múltiplas ${ }^{5}$.

Porém, deve-selevar em conta quea decisão é uma escolha entre duas ou mais opções ou alternativas. Quando uma pessoanão tem opção, não decide. Apenas adere a uma situaçãa ${ }^{31,33}$. Atualmente, a tendência do governo é reduzir os investimentos na rede hospitalar e transferir estes gastos para os programas de saúde da família e de cuidado domiciliar ${ }^{1,34}$.

Um dos grandes objetivos da assistência domiciliar é a busca da autonomia do paciente no processo de tomada de decisões sobre seu cuidado. No hospital, é o doente que se adapta ao meio; no domicílio, é o profissional que precisa se adaptar ${ }^{2,5,27}$. 0 atendimento domiciliar representa uma estratégia de atenção à saúde que engloba muito mais do que o simples fornecimento de um tratamento médico residencial; é um método aplicado ao cliente que enfatiza sua autonomia e esforça-se em real çar suas habilidades funcionais dentro de seu próprio ambiente ${ }^{2,35}$, visa proporcionar maior tranquilidade à família no cuidado de seu familiar doente ${ }^{3}$ e, principalmente, ajudar o cliente e a família a ter um estilo de vida saudável, independente da doença ou problema de saúde e de seu tratamento $0^{6,34,36}$.

A autonomia inclui a capacidade para a autodeterminação, para resistir a pressões sociais, para pensar e agir de maneira mais independente. A relação entre os membros de uma família, no cuidado domiciliar durante o processo da doença, é mantida, existe uma autonomia em ocupar o seu espaço, ou seja, cada um ocupa uma posição na estrutura familiar. Durante a hospitalização, os papéis estão predefinidos, uns são doentes, outros acompanhantes e os profissionais de saúde, os mandantes. Todos submetidos a regras elaboradas para garantir 0 andamento do serviço, o que nem sempre significa 0 melhor para quem está hospitalizado29. Um estudo realizado com pacientes hospitalizados re velou que o nível de autonomia dos mesmos quando internados era muito baixo, ficando os mesmos submissos às decisões de terceiros ${ }^{32,34}$.

A concorrência pela ocupação de leitos hospitalares e as filas de espera das instituições podem acarretar, por parte das famílias, uma insegurança quanto a uma possível reinternação. Cabe à equipe de saúde respeitar a decisão da família e proporcionar uma assistência adequada ao espaço e condições ou circunstâncias em que a situação se apresenta ${ }^{29}$.

Autonomia também deve ocorrer por parte dos profissionais, embora ainda seja pouco observada. Desta forma, os enfermeiros devem questionar o sistema de saúde e reivindicar recursos que respondam às necessidades dos pacientes, tendo estes direito ao cuidado: édever dos enfermeiros prestar esses cuidados ${ }^{37}$, tendo todo o respaldo legal, conforme a Lei $n^{0} 10.424^{31}$, já citada, ea Resolução do COFEN no 267/0138. Para a realização da assistência domiciliar à saúde, os profissionais apontam para a necessidade das práticas diferenciadas, mas ainda existem algumas dificuldades em sua implementação 29 .

\section{Discussão econclusões}

Ao refletir sobre as questões éticas no cuidado domiciliar, percebemos a necessidade das instituições de saúde e de seus profissionais proporcionarem ao pacientee cuidador um atendimento que realmente contemple as necessidades de saúde no domicílio.

As perspectivas relacionadas ao cuidado domiciliar são crescentes. Observa-se o interesse de muitos profissionais, bem como o incremento na formação de profissionais capacitados para abordar o planejamento, coordenação e avaliação das ações, a fim de constituir um processo contínuo de articulação com demais setores, cujas ações estejam relacionadas ao pacientee a família.

0 futuro aponta para novos espaços e novas relações de trabalho para a enfermagem no que 
se refere ao atendimento domiciliar, em que 0 trabalho empregado na prestação do cuidado deve ser revisado, bem como as formas contratuais exercidas atualmente.

Refletindo sobre os fatores que justificam o cuidado domiciliar e relacionando com os princípios éticos, como a beneficência, percebemos que a permanência hospitalar prolongada, e as re-hospitalizações contribuem para a elevação dos custos ao Sistema Único de Saúde eum maior risco ao paciente em contrair infecções nosocomiais. Assim, justifica-se a internação domiciliar, por se caracterizar em um cuidado que proporciona uma melhor qualidade de vida ao pacientee sua família.

Em contrapartida, a família inserida nesta ação de cuidado toma para si a responsabilidade que seria de um profissional. Assim, énecessário avaliar e respeitar as condições e limitações da família. Quando esta não consegue conviver com a presença de um paciente em casa, o cuidado domiciliar pode acarretar malefícios para o usuário e para a família.

A respeito do princípio da justiça, uma vez que as instituições de saúde visam à redução dos custos com internações e uma maior utilização de seus leitos, a indicação do cuidado domiciliar para alguns usuários apresenta-se como uma alternativa de assistência à saúde.

Por outro lado, cuidado domiciliar garante ao cliente condições de recuperação de sua saúde e continuidade do acompanhamento para além do hospital. Em decorrência da carência de recursos na área da saúde, o cuidado domiciliar se mostra como forma de garantir a atenção e atendimento às necessidades dos clientes.

É necessário questionar se estamos oferecendo o cuidado domiciliar como uma modalidade ou como a alternativa mais conveniente para a instituição e/ou sistema de saúde.

Diante de algumas reflexões, a capacidade de decidir por si mesmo, ou seja, a autonomia tanto do paciente como da família, para a opção do cuidado domiciliar, pode ser desrespeitada, pois deixa de ser uma opção e passa a ser uma imposição das instituições hospitalares.
E, por outro lado, estetipo de cuidado oportuniza a autonomia do paciente e da família no que se refere ao autocuidado no espaço domiciliar. Esta estratégia possibilita uma autonomia do cliente e da família, uma vez que o primeiro deixa de ser o doente e a família deixa de ser 0 acompanhante, passando todos a ocupar uma posição na estrutura familiar, ou seja, a mãecontinua sendo mãe, a filha, sendo filha.

Finalizamosqueo cuidado domiciliar deuma forma inovadora vem se tornando imprescindível para reduzir custos hospitalares, permanência hospitalar, número de reinternações e o número de complicações clínicas, bem como o aumento da participação da família no cuidado ao paciente, proporcionando uma melhor qualidade de vida. Entretanto, énecessário levar em conta a finali dade e qualidade desta assistência prestada, analisando-a sob a ótica dos princípios éti$\cos$, de modo a não tornar este tipo de cuidado benéfico apenas para uma das partes envolvidas esim um bem comum.

Os indicadores de avaliação usualmente utilizados são a adesão terapêutica por parte dos pacientes, a segurança na realização de cuidados no domicílio, a satisfação por parte do pacientee sua família com o atendimento fornecido, o grau de ansiedade com a situação vivenciada, a identificação de riscos, a participação da família no tratamento, 0 alcance do autocuidado, a adesão a ações de educação e saúde, o número de intercorrências e reinternações, a otimização do leito hospitalar e do atendimento ambulatorial e a redução de custos.

A valorização da família27 éuma atitude fundamental para estabelecer vínculo e confiança, como cumprir as combinações, sensibilizar-se com as situações encontradas no domicílio, estando abertos à flexibilização do plano.

Salienta-se ${ }^{3}$ que o cuidado realizado pelo enfermeiro no domicílio tem por objetivo a avaliação de indicadores derisco para infecção eorientações para o autocuidado ecuidado familiar frente às necessidades humanas básicas afetadas que influenciam no desenvolvimento das atividades de vida diárias. 


\section{Colaboradores}

LR Santos participou da concepção e desenho e da pesquisa bibliográfica, análise, interpretação e escrita do artigo; SS Funghetto e CGRM Ponce deLeon participaram da revisão crítica eda aprovação final do artigo.

\section{Referências}

1. Duarte YAO, Diogo MJE. Atendimento domiciliário: um enfoque gerontológico. In: Duarte YAO, Diogo MJE, organizadores. Atendimento domiciliar: um enfoque gerontológico. São Paulo: Atheneu; 2000. p. 3-17.

2. Santos BRL, Sagebin HV, Paskulin LMG, Eidt OR, Witt RR. O Domicílio como espaço do cuidado. In: Anais do $50^{\circ}$ Congresso Brasileiro de Enfermagem; 1998; Salvador. p. 121-132.

3. Beauchamp TL, Childress JF. Princípios de ética biomédica. São Paulo: Loyola; 2002.

4. Bardin L. Análise de conteúdo. Lisboa: Edições 70; 1979.

5. Mendes Junior WV. Um panorama da assistência domiciliar. Informe M éd-Lar [periódico na Internet]. 2001 [acessado 2008 mar 05]; 3. Disponível em: http://www.medlar.com.br/informes/06_2001/ panorama.asp

6. Cruz ICF, Barros SRTP, Ferreira HC. Enfermagem em Home Care e sua inserção nos níveis de atenção à saúde: a experiência da Escola de Enfermagem da Universidade Federal Fluminense. Enferm. atual. 2001; 1(4):35-38.

7. Paz AA. Subsídios para a estruturação de um programa de cuidado de enfermagem domiciliar ao paciente com sequelas neurológicas [monografia]. Porto Alegre (RS): Escola de Enfermagem, Universidade Federal do Rio Grande do Sul; 2002.

8. Cotta RM M , Suaréz-Varela M M , Cotta Filho JS, Llopis González A, Días Ricós JA, Real ER. La hospitalización domiciliaria ante los cambios demográficos y nuevos retos de salud. Rev Panam Salud Publica/Pan Am J Public Health 2002; 11(4): 253-261.

9. Martín AP, Perez Martin A, Rol De La Morena MJ, M areque Ortega M, Gomez Gomez M, Gomez Gomez C, Diaz De Cerio M. Efecto sobre el consumo de recursos hospitalarios de un programa de atención geriátrica domiciliaria en personas ancianas con patología cardiorrespiratoria muy evolucionada. Rev Esp Salud Pública 2001; 75(6): 559-567.

10. Papaléo-N eto $M$, Tieppo A. Serviço de assistência domiciliária da Clínica Geronto-Geriátrica do Hospital Servidor Público Municipal de São Paulo. In: Duarte YAO, Diogo MJE, organizadores. Atendimento domiciliar: um enfoque gerontológico. São Paulo: Atheneu; 2000. p. 549-563.

11. Jacob-Filho W, Chiba T, Andrade M V. Assistência domiciliária multiprofissional em uma instituição de ensino. In: Duarte YAO, Diogo MJE, organizadores. Atendimento domiciliar: um enfoque gerontológico. São Paulo: Atheneu; 2000. p. 539-544.

12. Martins JJ, Albuquerque GL, Nascimento ERP, Barra DCC, Souza WGA, Pacheco WNS. Necessidades de educação em saúde dos cuidadores de pessoas idosas no domicílio. Texto Contexto Enferm 2007; $16(2): 254-262$.

13. Williams A. What bothers caregivers of stroke victims? Jour N eurosc Nurs 1994; 26(3):155-161.

14. Canam C, Acorn S. Quality of life for family caregivers of people with chronic health problems. Rehab N urs 1999; 24(5):192-196. 
15. Perlini NMOG, Mancussi e Faro AC. Cuidar da pessoa incapacitada por acidente vascular cerebral no domicílio: o fazer do cuidador familiar. Rev Esc Enferm USP 2005; 39(2):154-163.

16. Machado ALG, Freitas CHA, Jorge MSB. 0 fazer do cuidador familiar: significados e crenças. Rev. Bras. Enferm. 2007; 60(5):530-534.

17. Fuente DO, Moreno SP, Rodríguez CM, López MR. Cuidados de enfermería en hospitalización a domicilio y hospitalización convencional. Rev Esp Salud Pública 1998; 72(6):517-527.

18. Brasil. Ministério da Saúde. Portaria $n^{\circ} 1.395$ de 9 de dezembro de 1999. Política Nacional do Idoso. [site da Internet] [acessado 2008 mar 10]. Disponível em: http://www.cofen.gov.br/resolucao.htm

19. Coyte PC, Young W. Regional variations in the use of home care services in Ontario, 1993/95. JAMC 1999; 161(4):376-380.

20. Duarte YAO, Jacob W Filho, Domingos MA, Izzo $H$. Programa de visita domiciliar do idoso - PROVIDI. In: Duarte YAO, Diogo MJE, organizadores. Atendimento domiciliar: um enfoque gerontológico. São Paulo: Atheneu; 2000.

21. Palombo T. Home care é alternativa para redução de custos com saúde - modelo de atendimento é priorizado para atender quadros crônicos. [site da Internet] 2008 [acessado 2008 mar 05]. Disponível em: http://www.doctorlife.com.br/artigo.asp?ld Artigo $=2$

22. Falcão HA. "Home Care" - uma alternativa ao atendimento da saúde. Revista Virtual da M edicina [periódico na Internet] 1999 jul-set [acessado 2008 mar 05]; 7(2). Disponível em: http://www.meonline.com. $\mathrm{br} / \mathrm{med}$ ed/med7/homecar.htm

23. Marin M JS, Caetano FB, Palasson RR. Avaliação da Satisfação dos U suários do Programa Interdisciplinar de Internação Domiciliar. Revista Espaço para a Saúde [periódico na Internet]. 2008 [acessado 2008 mar 05]. Disponível em: http://www.ccs.uel.br/ espacoparasaude/v2n2/doc/domiciliar.htm

24. Silva KL, Sena R, Leite JCA, Seixas CT, Gonçalves AM. Internação domiciliar no Sistema Único de Saúde. Rev. Saude Publica 2005; 39(3):391-397.

25. Creutzberg M. Vivências de famílias de classe popular cuidadoras de pessoas idosas fragilizadas: subsídios para o cuidado de enfermagem domiciliar [dissertação]. Porto Alegre (RS): Pós-Graduação em Enfermagem, Universidade Federal do Rio Grande do Sul; 2000.

26. Soto CCS, Acuña BLR, Hechavarría EC, Smith VVS. Aspectos bioéticos del ingreso domiciliario. Revista Cubana Enfermería 2002; 18(1):63-68.

27. Ribeiro VES. 0 domicilio como espaço par enfermagem: a experiência da enfermagem canadense. In: Anais do $50^{\circ}$ Congresso Brasileiro de Enfermagem; 1998; Salvador. p. 133-138.

28. Roubicek J, Salvatore A, Kavka G, Wiersba C. Servicio de internación domiciliaria para pacientes con enfermedades agudas. Rev Panam Salud Publica/ Pan Am J Public Health 1999; 6(3):172-176.

29. Marcelino SR, Radunz V, Erdmann AL. Cuidado Domiciliar: escolha ou falta de opção? Texto Contexto Enferm 2000; 9(3):9-21.
30. Giacomozzi CM, Lacerda MR. A prática da assistência domiciliar dos profissionais da estratégia de saúde da família. Texto Contexto Enferm 2006; $15(4): 645-653$.

31. Brasil. Lei 10.424 , de 15 de abril de 2002. Acrescenta capítulo e artigos à Lei 8080 de 19 de setembro de 1990, que dispõe sobre as condições de promoção, proteção e recuperação da saúde, a organização e funcionamento de serviços correspondentes e dá outras providências, regulamentando a assistência domiciliar no Sistema Único de Saúde. Diário Oficial da União 2002; 16 abr.

32. Brasil. Portaria 2.416, de 23 de março de 1998. Estabelece requisitos para credenciamento de hospitais e critérios para realização de Internação Domiciliar no SUS. Diário Oficial da União 1998; 23 mar.

33. Santos LR, Beneri RL, Lunardi VL. Questões éticas no trabalho da equipe de saúde: o (des) respeito aos direitos do cliente. Revista Gaúcha de Enfermagem 2005; 26(3):403-413.

34. Barros S. Home Care - palestra gera interesse em cursos de Home Care [site da Internet]. 2008 [acessado 2008 mar 05]. Disponível em: http://www. coren-rj.org.br/informes3.htm

35. Mantovani MF, Mottin JV Rodrigues J. Nurse Home Visit with Educational Activities in the arterial pressure treatment. Online Brazilian Journal of Nursing [pe riódico na Internet]. 2007 [acessado 2008 mar 05]; 6(1). Disponível em: http://www.uff.br/objnursing

36. Silva CAM, Acker JIBV. O cuidado paliativo domiciliar sob a ótica de familiares responsáveis pela pessoa portadora de neoplasia. Rev. Bras. Enferm. 2007; 60(2):150-154.

37. Pereira EGA, Costa MAM. Os centros de saúde em Portugal e o cuidado ao idoso no contexto domiciliário: estudo de um centro de saúde. Texto Contexto Enferm 2007; 16(3):408-416.

38. Brasil. Resolução COFEN № 267/01, de 05 de outubro de 2001. Aprova a atividades de Enfermagem em Domicílio Home Care [site da Internet] 2001 [acessado 2008 mar 05]. Disponível em: http://www.corenrj.org.br/site/resolucoes/RES_COFEN_267_2001.swf

Artigo apresentado em 05/12/2008

Aprovado em 26/06/2009

Versão final apresentada em 17/07/2009 